\title{
Accuracy of Three Electronic Apex Locators Compared with Digital Radiography: An Ex Vivo Study
}

\author{
Luigi Cianconi, MD, DDS, Vincenzo Angotti, DDS, Roberto Felici, DDS, Gabriele Conte, DDS, \\ and Manuele Mancini, DDS
}

\begin{abstract}
Ahstract
Introduction: This study compared (1) the accuracy of three different electronic apex locators (EALs) in detecting the apical foramen ex vivo under clinical conditions; (2) the accuracy of digital radiography and EALs in determining the working length (WL) with visible control under a microscope; and (3) the precision of \#10, \#15, and \#20 K-files in electronic measurements. Methods: The length of 101 extracted human teeth was measured with three different EALs (Endex [Osada Electric Co, Tokyo, Japan], ProPex II [Dentsply-Maillefer, Ballaigues, Switzerland], and Root ZX [J. Morita Co, Tustin, CA]), with radio videography (RVG) and compared with the actual length. An endodontic training kit (Pro-Train; Simit Dental, Mantova, Italy) was used during the experimental procedures. Results: Statistical analysis showed that Endex and ProPex II were more accurate than Root ZX in determining the WL. The paired sample $t$ test showed no statistically significant difference between the accuracy of the two radiographic planes examined. The $t$ test showed no significant difference between the three different K-file sizes measurements. Conclusions: Endex and ProPex II were more accurate than Root ZX in determining the actual WL. Instrument sizes of hand files did not affect the accuracy of EALs. EALs showed to be more accurate in determining the WL than RVG. (J Endod 2010;36:2003-2007)
\end{abstract}

\section{Key Words}

Electronic apex locator, Endex, ProPex II, radio videography, Root ZX, working length

From the Department of Restorative Dentistry and Endodontics, University of Rome "Tor Vergata," Rome, Italy.

Address requests for reprints to Dr Manuele Mancini, Via Tuscolana 58/64-00182 Rome, Italy. E-mail address: manuele. mancini@tiscali.it.

0099-2399/\$ - see front matter

Copyright (c) 2010 American Association of Endodontists. doi:10.1016/j.joen.2010.08.036
W orking length (WL) has been defined as "the distance from a coronal reference point to the point at which canal preparation and obturation should terminate" (1). It has been stated that the WL for instrumentation and obturation of the root canal system should be established at the apical constriction (2). The apical constriction, also referred to as a minor diameter, represents the transition between the pulpal and the periodontal tissue at the cement-dentinal junction. The cement-dentinal junction has been suggested as the position of termination of the canal filling (3). Anatomic studies have shown the apical constriction to be located 0.5 to $1.0 \mathrm{~mm}$ from the external or major foramen (2). Historically, methods of determining WL include the use of radiographs, tactile sensation, and electronic apex locators (EALs). However, radiographs are subject to distortion, magnification, interpretation variability, and lack of threedimensional representation. The magnification error has been found to be $5.4 \%$ with a paralleling technique (4). As a result, WL determined from radiographs is generally measured about 0.5 to $1 \mathrm{~mm}$ short of the radiographic apex. Pratten and McDonald (5) showed that the assumption of the apical constriction being $1 \mathrm{~mm}$ short of the radiographic apex will result in an underestimation of WL. Vertical and horizontal cone angulations, film processing issues, tooth inclination, and film position will influence WL determination from radiographs (6). In some teeth, the major foramen may be located up to $3.5 \mathrm{~mm}$ from the radiographic apex (7). In such teeth, if the canal terminates in the plane of the film, the radiographic appearance will be "short," and any adjustment will result in the WL being overextended. A WL established beyond the minor diameter may cause apical perforation and overfilling of the root canal system. This may increase postoperative pain and delay or prevent healing. Alternately, a WL established short of the minor diameter may lead to inadequate debridement and underfilling of the canal. However, tactile sense is quite variable, and accuracy is questionable (8). Root canals with excessive curvature, an immature apex, or calcified canals will hinder the tactile sensation of the apical constriction. Custer (9) was the first to introduce an electrical method of locating the apical foramen. Suzuki's (10) discovery that electrical resistances between the periodontal ligament and oral mucosa registered constant values of $6.5 \mathrm{k} \Omega$ led to the development of the first EAL (Sunada) (11). The accuracy of EALs was poor because of the influence of fluids or pulp tissue in the canal. Advances in EAL technology have led to the development of EALs that make accurate readings in the presence of electrolytes. Manufacturers suggest that canals be moist rather than dry if more accurate WL readings are to be achieved. Özsezer et al (12) found that the WL measurements with the Propex (Dentsply-Maillefer, Ballaigues, Switzerland) were more accurate after extirpation of the pulp than after the use of irrigation solutions, among which the accuracy of WL determination was highest with chlorhexidine gluconate followed by sodium hypochlorite and saline. Endex (Osada Electric Co, Tokyo, Japan) was created in 1984 by Yamaoka based on studies by Ushyama (13). It is based on the difference in impedance between two wavelengths ( 1 and $5 \mathrm{kHz}$ ). The Endex can measure WL more precisely in wet root canals (14) than in dry root canals (15). Moreover, Endex must be calibrated for each canal. Many studies (16-18) have shown precision measurements $\pm 0.5 \mathrm{~mm}$ from the $\mathrm{WL}$ in $59 \%, 100 \%$, and $68 \%$ of the specimens treated. Advances in technology have led to the development of EALs such as the Root ZX (J. Morita Co, Tustin, CA) that determine the minor diameter position using the "ratio method," which allows for the simultaneous measurement of impedance at two frequencies; a quotient of impedance is then calculated and expressed as a position of the file in the canal. The Root ZX works in the presence of electrolytes 


\section{Basic Research-Technology}

and nonelectrolytes and requires no calibration (19). In vivo studies have shown the Root ZX to be accurate in locating the minor diameter to within $1 \mathrm{~mm}$ (20-23). Recently, a new EAL, the ProPex II, has been developed (Dentsply-Maillefer). It measures with multisignal frequencies the energy of the signal, not the amplitude as for all EALs.

The purpose of this study was three-fold: (1) to compare the accuracy of Endex, Root ZX, and ProPex II in detecting the apical foramen $e x$ vivo under clinical conditions; (2) to compare the accuracy of digital radiography and EALs in determining the $\mathrm{WL}$, with visible control under a microscope; and (3) to compare the precision of \#10, \#15, and \#20 $\mathrm{K}$-files in electronic measurements.

\section{Materials and Methods}

We selected 101 periodontally involved human teeth extracted from 35- to 60-year-old patients, with the approval of the Ethics in Research Committee of the Centre of Health Sciences of the University of Rome "Tor Vergata." After being cleaned, each root was carefully examined by stereomicroscopy (Universal-300; Moeller-Wedel, Wedel, Germany) at $20 \times$ magnification for the detection of the presence of external cracks, wide-open apices, or apices undergoing resorption, which might alter the accuracy of the WL measurements. The teeth were devoid of caries, endodontic treatments, or restorations. After extraction, teeth were stored in $2 \%$ thymol solution at room temperature and used within 1 week. Each crown was sectioned at midlevel by means of a crosscut carbide bur (Dentsply-Maillefer) in a highspeed handpiece with water spray to produce a flat surface for the precise location of the rubber stop. Endodontic access to the pulp chamber was gained with a round diamond-coated bur (Dentsply-Maillefer). Pulp canal debris was removed from the coronal third of the canal with a \#4 Gates-Glidden bur. After each canal was identified, three different K-files (\#06, \#08, and \#10; Dentsply-Maillefer) were passed through the apex to verify canal patency. Excess fluid was removed from the pulp chamber, but no attempt was made to dry the canal. The specimens were placed in a tooth holder, which is part of an endodontic training kit (Pro-Train; Simit Dental, Mantova, Italy) (Fig. 1). The conducting medium was an ecoelectroconductive gel (Farmacare Srl, Casale, Italy). The gel was poured into the box before the specimen was inserted into the endodontic training kit. The specimen was tightly held inside the tooth holder in the lid of the Pro-Train to prevent displacement during measurements.

\section{EAL Measurements}

We used three different K-files (\#10,\#15, and \#20) with a silicone stop to make multiple electronically determined $\mathrm{WL}$ readings in each

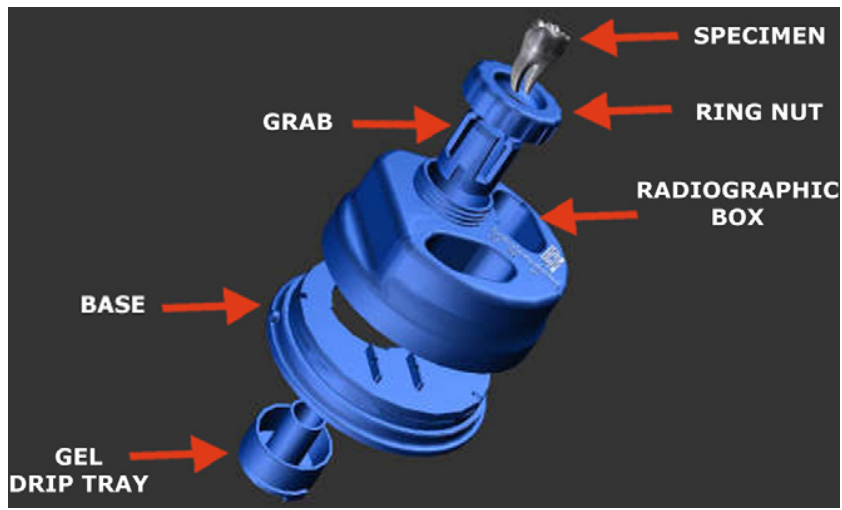

Figure 1. Pro-Train and its components. (This figure is available in color online at www.aae.org/joe/.) canal using the three EALs. Because three EALs were used with each canal, we alternated the first EAL to be used in each successive canal. After recording the WL with the first EAL, we withdrew the \#10 file and measured with a digital caliper (Mitutoyo 571-202-20; Mitutoyo Italiana SRL, Linate, Italy), which was recorded to the nearest 0.5 $\mathrm{mm}$. After recording the $\mathrm{WL}$, we reinserted the same file to determine the WL using the second and third EALs and measuring in the same manner. The same procedure was repeated for each specimen with the K-files \#15 and \#20. For each specimen, we obtained nine electronic measures (three for each K-file). To reduce variables, only one calibrated operator carried out the electronic readings with endpoints indicated. For the Endex, the file was advanced until the analog numeric bar read 0.0 within the red interval. For the Root $Z X$, the file was advanced until the liquid crystal display indicated the flashing word "APEX." For the ProPex II, the file was advanced until the liquid crystal display indicated the flashing word "APEX" and " 0.0 ," indicating the location of the foramen (according to the manufacturer's instructions).

\section{Digital Radiography Measurements}

We used average electronic measurements to take radiographs. We used the K-file that showed the mean of measurements closest to the average of the nine electronic measurements taken for each specimen. If the three files showed the same electronic measurement mean, we used the largest. We made radiographic measurements with a $0^{\circ}$ inclination in both the mesiodistal and buccolingual planes using the Pro-Train radio videography sensor holders and a custom base where Pro-Train, an RVG sensor (DSX Digital Sensor; Anthos Impianti SRL, Imola, Italy), a radiographic tube (Evolution X 3000; New Life Technology SRL, Grugliasco, Italy), and specimens were firmly blocked and collimated (Fig. 2). The distance from the end of the file and the radiographic apex was measured (Image Easy Managing, Anthos Impianti SRL) and recorded. A consensus in the measurements was always reached among three investigators after examining 20 specimens jointly for calibration purposes. Intra- and interexaminer reliability for RVG assessment was verified by the Kappa test.

\section{Actual WL Measurements}

After the RVG analysis, we removed the specimen from the socket of the Pro-Train, ensuring that it had not been displaced during the electronic and RVG measurements. We measured the root length (actual

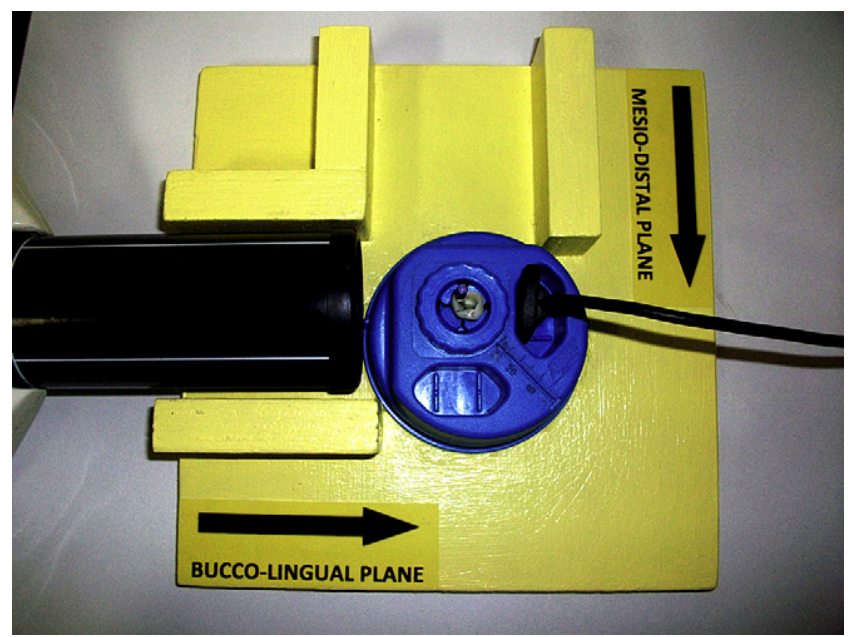

Figure 2. Experimental apparatus during radiographic measurements. (This figure is available in color online at www.aae.org/joe/.) 
Basic Research-Technology

TABLE 1. Statistical Analysis on the Accuracy of EALs and the Actual Working Length (mm)

\begin{tabular}{|c|c|c|c|c|c|c|}
\hline & Mean & Standard Deviation & Minimum & Maximum & $t$ Value & $\operatorname{Pr}>[t]$ \\
\hline $\operatorname{ENDEX}(n=101)$ & 0.23 & 0.34 & $-0.5^{\star}$ & 1 & 7.03 & $p<0.001$ \\
\hline ROOT ZX $(n=98)$ & 0.50 & 0.42 & $-0.17 *$ & 1.67 & 11.88 & $p<0.001$ \\
\hline PROPEX $\|(n=101)$ & 0.27 & 0.37 & $-0.67 *$ & 1.5 & 7.49 & $p<0.001$ \\
\hline
\end{tabular}

EAL, electronic apex locator.

*Negative values indicate that the K-file's tip is inside the root canal.

WL) using the same K-file used for the RVG measurement. Using a microscope at $5 \times$ magnification (Carl Zeiss $\mathrm{GmbH}$, Oberkochen, Germany), we inserted the K-file with a silicone stop into the root until its tip was observed at the level of the most coronal border of the apical foramen. When the file tip was observed at the apical foramen, the stop was stabilized at the coronal edge of the tooth, the file was removed, and the distance between the stop and the file tip was measured with a digital caliper. Blind evaluation was performed independently by three observers after examining 20 specimens jointly for calibration purposes. Intra- and interexaminer reliability for actual WL assessment was verified by the Kappa test. We recorded a mean value of actual WL measurements for each tooth and subjected it to statistical analysis by the paired $t$ test to compare the numbers of teeth with precise measurements for each apex locator. We also used the paired $t$ test to compare the measurements of the apex locators for each tooth because the same teeth were used. We used the same statistical analysis to determine the accuracy of the radiographic method and to compare the accuracy of the 3 K-files.

\section{Results}

Kappa test results, with a significance set at 0.5 , showed good intra- and interexaminer agreement, with values ranging from 0.90 and above for the different groups. EAL accuracy values are shown in Table 1. Endex and ProPex II were more accurate than Root ZX in determining the WL. The mean differences between EAL WL measurements and actual WL (Endex, Root ZX, and ProPex II) were $0.23 \mathrm{~mm}, 0.50$ $\mathrm{mm}$, and $0.27 \mathrm{~mm}$, respectively. The apical foramen $( \pm 0.5 \mathrm{~mm})$ was determined in $84.1 \%, 62.4 \%$, and $82.2 \%$ of the specimens for Endex, Root ZX, and ProPex II, respectively. All EALs showed the following significant tendency toward overestimation: $15.9 \%, 37.6 \%$, and $17.8 \%$, respectively, for Endex, Root ZX, and ProPex II. No measures were underestimated. The radiographic position of the file's tip was consistent with the apical foramen in $44.5 \%$ and $51.5 \%$ of the specimens in the buccolingual and mesiodistal planes, respectively, with a significant tendency toward underestimation. The mean distances between the file tip and the radiographic apex were $0.02 \mathrm{~mm}$ $( \pm 0.42)$ and $0.05 \mathrm{~mm}( \pm 0.39)$, respectively, for the buccolingual and the mesiodistal planes (Table 2). No electronic measurements were influenced by the three different instrument sizes of hand files.

\section{Statistical Analysis}

We used a paired sample $t$ test to compare the results and determined a significant difference at a $99.9 \%$ confidence level. The analysis was performed with the SAS System (SAS Institute SRL, Milan, Italy). Statistical analysis showed a significant difference between Root ZX $(p<0.001)$ and both ProPex II and Endex measurements. There was no statistically significant difference between ProPex II and Endex. The $t$ test showed no statistically significant difference between the accuracy of the two radiographic planes examined. The $t$ test showed no significant difference between the three different sizes of K-files.

\section{Discussion}

Numerous studies have reported on the accuracy of EALs for determining the location of the minor diameter $(21,22,24)$. EALs can accurately determine the WL in $75.0 \%$ to $96.5 \%$ of the root canals with mature apices (25-27). These studies differ in establishing the reference point from which measurement accuracy is determined. Some authors measured from the minor diameter (apical constriction) (21, 22, 27-33), whereas others measured from the major diameter or apical foramen $(16,23,34)$. EALs have traditionally afforded some latitude of acceptable error in locating the apex $(21,25,26,30)$. As a result, different ranges have been used in the evaluation of EAL accuracy. Diverse studies have usually considered the electronic measurements for the minor constriction to be between the $0.5-\mathrm{mm}$ mark $(16,20,21,28-30,32,33,35$, $36)$, which is considered highly accurate (37), and the 1-mm mark $(24,36,38-40)$. One reason cited for accepting a 1.0-mm margin of error is the wide range seen in the shape of the apical third (21, 41). Thus, this variation is clinically acceptable (21) because microscopic studies revealed that this landmark might be positioned within this range $(2,41,42)$. Nevertheless, whatever the apical limit, the measuring device used should be precise and reliable (ie, able to locate the chosen limit and giving similar readings when used by more than one operator [37]). The materials most often used are alginate $(28,31,32,35,40)$, agar $(36,43)$, saline solution $(24,27)$, and gelatin (44). The validity of measurements made with in vitro models (ie, the extent to which they depict the clinical accuracy of EALs) is unknown (36). However, they are able to reproduce the clinical condition of EAL use and facilitate the objective examination of several

TABLE 2. Statistical Analysis on the Accuracy of EALs and RVG System Measurements (mm)

\begin{tabular}{|c|c|c|c|c|c|c|}
\hline Buccolingual Plane & Mean & Standard Deviation & Minimum & Maximum & $t$ Value & $\operatorname{Pr}>[t]$ \\
\hline ENDEX $(n=99)$ & 17.27 & 2.24 & 12.1 & 21.6 & 76.48 & $p<0.001$ \\
\hline ROOT ZX $(n=96)$ & 17.40 & 2.25 & 12.1 & 22.07 & 75.78 & $p<0.001$ \\
\hline PROPEX II $(n=99)$ & 17.25 & 2.29 & 12.1 & 21.6 & 74.89 & $p<0.001$ \\
\hline Mesiodistal Plane & Mean & Standard Deviation & Minimum & Maximum & $t$ Value & $\operatorname{Pr}>[t]$ \\
\hline $\operatorname{ENDEX}(n=101)$ & 17.19 & 2.20 & 12.1 & 21.5 & 78.32 & $p<0.001$ \\
\hline ROOT ZX $(n=98)$ & 17.36 & 2.20 & 12.1 & 22.07 & 77.99 & $p<0.001$ \\
\hline PROPEX II $(n=101)$ & 17.22 & 2.26 & 12.1 & 21.5 & 76.57 & $p<0.001$ \\
\hline
\end{tabular}

EAL, electronic apex locator; RVG, radio videography. 
TABLE 3. Resistance Values $(\mathrm{k} \Omega)$ of Alginate Mixed with Deionized Water or Tap Water and the Ecoelectroconductive Gel

\begin{tabular}{lccc}
\hline & $\mathrm{T}=\mathbf{0} \mathrm{min}$ & $\mathrm{T}=1 \mathrm{~min}$ & $\mathrm{~T}=5 \mathrm{~min}$ \\
\hline $\begin{array}{l}\text { Alginate with } \\
\text { deionized water }\end{array}$ & $20 \mathrm{~K} \Omega$ & $415 \mathrm{~K} \Omega$ & $1035 \mathrm{~K} \Omega$ \\
$\begin{array}{l}\text { Alginate with tap } \\
\text { water }\end{array}$ & $15 \mathrm{~K} \Omega$ & $220 \mathrm{~K} \Omega$ & $843 \mathrm{~K} \Omega$ \\
Gel & $255 \mathrm{~K} \Omega$ & $255 \mathrm{~K} \Omega$ & $255 \mathrm{~K} \Omega$ \\
\hline
\end{tabular}

variables that is not practical in in vivo studies $(36,43)$. It has been suggested that EALs operate on the principle of electricity rather than the biological properties of the tissues involved (45). Therefore, models in which extracted teeth are immersed in media with electrical resistance similar to that of the periodontal tissue can give precise and reliable information on their function (43). In the current study, an ecoelectroconductive gel was used as a medium because of its suitable electroconductive property. The resistance of alginate mixed with deionized water or tap water and the ecoelectroconductive gel was tested (Table 3). Results showed that gel was stable with time and not influenced by room temperature and humidity compared with both alginate mixtures. In fact, 5 minutes from the end of mixing, alginate resistivity was increased 10-fold compared with the first minute after mixing and five-fold higher than gel. ProTrain and gel poured into a dedicated box constitute an effective tool for the evaluation of EALs and familiarize the operator with electronic root canal length measurement because of its high degree of stability, low cost, and simplicity of execution. Furthermore, the roots clamped by the ProTrain holder are sufficiently strongly held to resist the force exerted by experimental procedures. It also allows for the roots to be hidden, making it possible for measurements to be made objectively with minimum bias. We did not use $\mathrm{NaOCl}$ throughout the study to avoid denaturing root dentin during repeated measurements on the same specimens; therefore, we used $\mathrm{NaOCl}$ initially to help eliminate root canal debris but not thereafter. In the current study, to reduce variables according to the operator's manual, only one calibrated operator carried out the electronic readings, which was essential to obtaining precise and consistent results. In this study, the mean distance beyond the minor diameter was $0.3 \mathrm{~mm}$, with a range of $-0.5 \mathrm{~mm}$ to $1 \mathrm{~mm}$. Different ex vivo methods have been used to investigate the accuracy of EALs. One is shaving the apical portion of the root along the long axis of the tooth in a plane determined to show the best representation of the minor diameter in relation to the file. If the apical portion is not shaved, the relationship between the file tip and the constriction cannot be established. In the current study, this method was not used because the choice of the "APEX" mark on the display led to the use of the apical foramen as a landmark, rendering the shaving of the apical portion of the root unnecessary. To compare EAL accuracy, we also used radiographic analysis. A problem with radiographic comparisons in vivo is that the anatomic foramen can be located anywhere from 0.0 to $3.0 \mathrm{~mm}$ from the radiographic apex (2). Pineda and Kuttler (46) reported the foramen to be located away from the anatomic apex in $83 \%$ of teeth examined. Because conditions similar to those in the clinic would not be as ideal because of magnification errors and overestimation of canal length, in this study $28.5 \%$ of the teeth appearing radiographically acceptable actually had the file tip beyond the foramen. The results of this study are in agreement with those of previous studies in which 15 of the 22 files (68.2\%) that appeared on buccolingual projection to be at or short of the radiographic apex actually had the file tip beyond the desired WL. These results are in agreement with those of other studies (20). In vitro, EALs were $10 \%$ to $40 \%$ more accurate than radiographs in locating the minor diameter $(5,18)$. Also of interest was the relative size of the file compared with the diameter of the apical endpoint. Manufacturers of EALs recommend using the largest file that registers a WL reading. Briseño-Marroquin et al (47) studied the accuracy of four different EALs with three different instrument sizes of hand files. They found that sizes \#08, \#10, and \#15 had no influence on the accuracy of WL determination in agreement with our results. In vitro studies have shown the Root ZX to be $62.7 \%$ to $68 \%$ accurate to within $0.5 \mathrm{~mm}$ and $90 \%$ to $97.5 \%$ accurate to within $1 \mathrm{~mm}$ of the minor diameter (18, $24,48)$. The accuracy of the Endex, ProPex II, and Root ZX in locating the apical foramen within $0.5 \mathrm{~mm}$ was $86.1 \%, 83.2 \%$, and $65.3 \%$, respectively, with mean distances of $0.31,0.45$, and $0.57 \mathrm{~mm}$, respectively, past the minor diameter. Siu et al (49) showed in an in vitro study that the mean distance to the minor diameter was between 0.16 and $0.22 \mathrm{~mm}$. Lucena-Martin et al (35), testing the in vitro accuracy of 3 EALs in vitro, showed that in 5\% of the canals the measurements surpassed the apical foramen. These results are in agreement with those of the present study in which the file tip was beyond the apical foramen in $51.4 \%$ of the canals when the "APEX" mark on the dial was used. This fact must be seriously considered because in clinical conditions, in contrast to in vitro studies, a greater variation in measurements is expected because the favorable circumstances for precise measurements are not available (39), and, consequently, an overestimated WL could lead to a poor prognosis (50). These findings raise the question of whether the WL should be established at the point where the EAL indicates the apical foramen or at some distance coronal to that point (33). Mayeda et al (51) suggest that "each operator should correlate his own radiographic and clinical findings with the analog dial readings on the instrument to determine exactly where on the dial the operator wants to call his apex." In this way, some authors have proposed withdrawing the instrument 0.5 or $1 \mathrm{~mm}$ when using the Root ZX with the display meter setting at " 0.5 " to ensure that the file tip does not protrude beyond the apical constriction, avoiding root canal overpreparation $(23,30)$.

\section{Conclusions}

Based on the results of the present study, to prevent overestimation of the root canal length using the three different EALs tested, $1 \mathrm{~mm}$ should be subtracted from the measurement on the "APEX" mark. EALs have shown to be more precise than digital radiography in detecting the apical foramen. Electronic measurements were not influenced by instrument sizes of hand files.

\section{Acknowledgment}

We would like to thank Dr Marco Costantini from the Chiesi Group SpA (Parma, Italy) for assisting with the statistical analysis.

\section{References}

1. Glossary of Endodontic Terms. 7th ed. Chicago, IL: American Association of Endodontists; 2003.

2. Kuttler Y. Microscopic investigation of root apexes. J Am Dent Assoc 1955;50: 544-52.

3. Grove CJ. A new simple standardized technique producing perfect fitting impermeable root canal fillings extended to the dento-cemento junction. Dent Items Interest 1928;50:855-7.

4. Vande Voorde HE, Bjorndahl AM. Estimating endodontic "working length" with paralleling radiographs. Oral Surg Oral Med Oral Pathol Oral Radiol Endod 1969;27: 106-10.

5. Pratten $\mathrm{DH}$, McDonald NJ. Comparison of radiographic and electronic working lengths. J Endod 1996;22:173-6.

6. Goldman M, Pearson AH, Darzenta N. Endodontic success: who's reading the radiograph? Oral Surg Oral Med Oral Pathol Oral Radiol Endod 1972;33:432-7. 
7. Green DA. Stereo binocular microscopic study of the root apices and surrounding areas of 100 mandibular molars. Oral Surg Oral Med Oral Pathol Oral Radiol Endod $1955 ; 8: 1298$.

8. Seidberg BH, Alibrandi BV, Fine $\mathrm{H}$, et al. Clinical investigation of measuring working lengths of root canals with an electronic device and with digital-tactile sense. J Am Dent Assoc 1975;90:379-87.

9. Custer LE. Exact methods of locating the apical foramen. Q Natl Dent Assoc 1918;5: $815-9$

10. Suzuki K. Experimental study on iontophoresis. J Jap Stomatol 1942;16:411.

11. Sunada I. New method for measuring the length of the root canal. J Dent Res 1962 41:375-87.

12. Özsezer E, Inan $\mathrm{U}$, Aydin $\mathrm{U}$. In vivo evaluation of ProPex electronic apex locator. J Endod 2008;33:974-7.

13. Ushiyama J. New principle and method for measuring the root canal length. J Endod 1983;9:97-104

14. Saito T, Yamashita Y. Electronic determination of root canal length by newly developed measuring device-influence of the diameter of apical foramen, the size of $\mathrm{K}$ file and the root canal irrigant. Dent Jpn (Tokyo) 1990;27:65-72.

15. Frank AL, Torabinejad M. An in vivo evaluation of Endex electronic apex locator. J Endod 1993;19:177-9.

16. Weiger R, John C, Geigle H, et al. An in vitro comparison of two modern apex locators. J Endod 1999;25:765-8.

17. De Moor RJ, Hommez GM, Martens LC, et al. Accuracy of four electronic apex locators: an in vitro evaluation. Endod Dental Traumatol 1999;15:77-82.

18. Martinez-Lozano MA, Forner-Navarro L, Sanchez-Cortés JL, et al. Methodological considerations in the determination of working length. Int Endod J 2001;34:371-6.

19. Kobayashi C, Suda H. New electronic canal measuring device based on the ratio method. J Endod 1994;20:111-4.

20. Welk AR, Baumgartner JC, Marshall JG. An in vivo comparison of two frequencybased electronic apex locators. J Endod 2003;29:497-500.

21. Shabahang S, Goon WWY, Gluskin AH. An in vivo evaluation of Root ZX electronic apex locator. J Endod 1996;22:616-8.

22. Dunlap CA, Remeikis NA, BeGole EA, et al. An in vivo evaluation of an electronic apex locator that uses the ratio method in vital and necrotic canals. J Endod 1998;24: $48-50$.

23. Pagavino G, Pace R, Baccetti T. An SEM study of in vivo accuracy of the Root ZX electronic apex locator. J Endod 1998;24:438-41.

24. Goldberg F, De Silvio AC, Manfre S, et al. In vitro measurement accuracy of an electronic apex locator in teeth with simulated apical root resorption. J Endod 2002;28: $461-3$.

25. Gordon MP, Chandler NP. Electronic apex locators. Int Endod J 2004:37:425-37.

26. Nekoofar MH, Ghandi MM, Hayes SJ, et al. The fundamental operating principles of electronic root canal length measurement devices. Int Endod J 2006;39:595-609.

27. El Ayouti A, Weiger R, Lost C. The ability of root ZX apex locator to reduce the frequency of overestimated radiographic working length. J Endod 2002;28:116-9.

28. D'Assunção FL, de Albuquerque DS, Salazar-Silva JR, et al. The accuracy of root canal measurements using the Mini Apex Locator and Root ZX-II: an evaluation in vitro. Oral Surg Oral Med Oral Pathol Oral Radiol Endod 2007;104:e50-3.

29. Wrbas KT, Ziegler AA, Altenburger MJ, et al. In vivo comparison of working length determination with two electronic apex locators. Int Endod J 2007;40:133-8.

30. Haffner C, Folwaczny M, Galler K, et al. Accuracy of electronic apex locators in comparison to actual length—an in vivo study. J Dent 2005;33:619-25.
31. Herrera M, Abalos C, Planas AJ, et al. Influence of apical constriction diameter on Root ZX apex locator precision. J Endod 2007;33:995-8.

32. Plotino G, Grande NM, Brigante L, et al. Ex vivo accuracy of three electronic apex locators: Root ZX, Elements Diagnostic Unit and Apex Locator and ProPex. Int Endod J 2006;39:408.

33. Tselnik M, Baumgartner JC, Marshall JG. An evaluation of Root ZX and Elements Diagnostic apex locators. J Endod 2005;31:507-9.

34. Guise GM, Goodell GG, Imamura GM. In vitro comparison of three electronic apex locators. J Endod 2010;36:279-81.

35. Lucena-Martin C, Robles-Gijon V, Ferrer-Luque CM, et al. In vitro evaluation of the accuracy of three electronic apex locators. J Endod 2004;30:231-3.

36. Ebrahim AK, Wadachi R, Suda H. Ex vivo evaluation of the ability of four different electronic apex locators to determine the working length in teeth with various foramen diameters. Aust Dent J 2006;51:258-62.

37. Ounsi HF, Naaman A. In vitro evaluation of the reliability of the Root ZX electronic apex locator. Int Endod J 1999;32:120-3.

38. Goldberg F, Marroquin BB, Frajlich S, et al. In vitro evaluation of the ability of three apex locators to determine the working length during retreatment. J Endod 2005; 31:676-8.

39. El Ayouti A, Kimionis I, Chu AL, et al. Determining the apical terminus of root-end resected teeth using three modern apex locators: a comparative ex vivo study. Int Endod J 2005;38:827-33.

40. Bernardes RA, Duarte MA, Vasconcelos BC, et al. Evaluation of precision of length determination with 3 electronic apex locators: Root ZX, Elements Diagnostic Unit and Apex Locator, and RomiAPEX D-30. Oral Surg Oral Med Oral Pathol Oral Radiol Endod 2007;104:e91-4.

41. Dummer PM, McGinn JH, Rees DG. The position and topography of the apical canal constriction and apical foramen. Int Endod J 1984;17:192-8.

42. Ricucci D. Apical limit of root canal instrumentation and obturation, part 1. Literature review. Int Endod J 1998;31:384-93.

43. Ebrahim AK, Wadachi R, Suda $\mathrm{H}$. In vitro evaluation of the accuracy of five different electronic apex locators for determining the working length of endodontically retreated teeth. Aust Endod J 2007;33:7-12.

44. Jenkins JA, Walker WA 3rd, Schindler WG, et al. An in vitro evaluation of the accuracy of the root ZX in the presence of various irrigants. J Endod 2001;27:209-11.

45. Huang L. An experimental study of the principle of electronic root canal measurement. J Endod 1987;13:60-4.

46. Pineda F, Kuttler Y. Mesiodistal and buccolingual roentgenographic investigation of 7,275 root canals. Oral Surg Oral Med Oral Pathol Oral Radiol Endod 1972;33: 101-10.

47. Briseño-Marroquin B, Frajlich S, Goldberg F, et al. Influence of instrument size on the accuracy of different apex locators: an in vitro study. J Endod 2008;34:698-702.

48. de Camargo EJ, Zapata RO, Medeiros PL, et al. Influence of preflaring on the accuracy of length determination with four electronic apex locators. J Endod 2009;35: $1300-2$.

49. Siu C, Marshall JG, Baumgartner JC. An in vivo comparison of the Root ZX II, the Apex NRG XFR, and Mini Apex Locator by using rotary nickel-titanium files. J Endod 2009;35:962-5.

50. Naito T. Better success rate for root canal therapy when treatment includes obturation short of the apex. Evid Based Dent 2005;6:45.

51. Mayeda DL, Simon JH, Aimar DF, et al. In vivo measurement accuracy in vital and necrotic canals with the Endex apex locator. J Endod 1993;19:545-8. 